\title{
Systematic Review of Emerging Models of Cancer Care: Implications for the Health Industry
}

\author{
Bita Kash ${ }^{1^{*}}$, Molly McKahan ${ }^{2}$, Sarah Mack ${ }^{2}$ and Upali Nanda ${ }^{3}$ \\ ${ }^{1}$ Department of Health Policy \& Management School of Public Health, Texas A\&M University, USA \\ ${ }^{2}$ NSF Center for Health Organization Transformation School of Public Health, Texas A\&M University \\ ${ }^{3}$ Center for Advanced Design Research and Evaluation, HKS, Inc., USA
}

*Corresponding author: Bita Kash, Associate Professor, Department of Health Policy \& Management School of Public Health, Texas A\&M University, USA, Tel: + 979 575 6768; E-mail: bakash@sph.tamhsc.edu

Received Date: July 12, 2017; Accepted Date: July 28, 2017; Published Date: August 07, 2017

Copyright: ( 2017 Kash B, et al. This is an open-access article distributed under the terms of the Creative Commons Attribution License, which permits unrestricted use, distribution, and reproduction in any medium, provided the original author and source are credited.

\begin{abstract}
In selected patients diagnosed with Breast Cancer (BC), adjuvant chemotherapy might reduce local and systemic recurrence risk, as well as cancer death rate. The combination of Docetaxel and Cyclophosphamide (TC) is a wellrecognized effective adjuvant chemotherapy regimen. Nonetheless, a considerable high rate of febrile neutropenia (FN) is associated with this regimen. We sought to investigate hematologic toxicity associated with adjuvant TC in a non-selected, "real world" cohort of BC patients.
\end{abstract}

Methods: We reviewed the electronic medical records of patients who presented to the Oncology Center from Hospital Sírio-Libanês (HSL) and Instituto do Câncer do Estado de Sao Paulo (ICESP). Patients included in the analysis received adjuvant chemotherapy with TC regimen after definitive breast surgery.

Results: 95 patients with were included in our analysis. Median age was 55.5 years. All patients had a good performance status (either ECOG 0 or 1), and the great majority had no comorbidities. Most patients received 4 cycles of chemotherapy (80\%). Data on granulocyte colony stimulating factor (G-CSF) administration was available in 85 patients from our cohort. G-CSF was used as primary prophylaxis in 31 patients, and as secondary prophylaxis in 13 patients, following a prior episode of febrile neutropenia. Overall, fifteen women (15.8\%) had a documented FN episode. Among women who received G-CSF as primary prophylaxis, the rate of FN was $6.45 \%$ (2 patients). In contrast, among patients who did not receive primary prophylaxis with G-CSF, FN rate was considerably higher, namely $24.07 \%$ (13 patients). Patients who received primary prophylaxis with G-CSF had a statistically significant lower risk of experiencing a FN episode $(p=0.049)$.

Conclusion: Febrile Neutropenia rate in this group of non-selected BC patients was higher than previous reported on randomized controlled trials that evaluated adjuvant TC regimen in the same dosing and schedule as used in our cohort. Primary prophylaxis with G-CSF was associated with a statistically significant lower risk of FN and should be considered in the management of patients who receive this chemotherapy combination.

Keywords: Cancer care; Emerging models; Facility implications

\section{Introduction}

Currently 15.5 million Americans with a history of cancer are living in the United States [1], including both persons with ongoing diagnoses and in remission. Approximately 1.6 million new cancer cases are diagnosed annually with nearly 600,000 cancer related deaths, which places cancer as the second most common cause of death in the United States, next to heart disease [2]. While 32\% of all cancer deaths in the US are attributed to cigarette smoking, other risk factors include body fatness, physical inactivity, excess alcohol consumption, poor diet/nutrition, infectious agents (i.e. HPV, HBV, HCV, HIV, and $H$. pylori), genetics, stress, and environmental exposures (i.e. carcinogenic compounds and UV radiation) [2,3]. The lifetime risk of developing cancer is $42 \%$ in men and $38 \%$ in women, with the most common cancers including lung, colorectal, breast, and prostate [2]. People 50 years of age or older account for $86 \%$ of all cancer diagnoses [2].
There are several major trends in cancer prevalence within the United States: new diagnosis rates have increased due to the aging population, overall incidence (per 100,000 persons) has decreased for most cancers, and incidence-mortality has dropped. The combination of these conditions is resulting in an increase in both cases of cancer and survivorship. Cancer related mortality dropped by $23 \%$ from its peak in 1991 to 2012 due to reductions in smoking and improvements in early detection and treatment [2]. The 5-year survival rate for all cancers has increased from $49 \%$ in 1977 to $69 \%$ in 2011 [2]. Survivorship includes many status trajectories, including complete cancer eradication, late complications of treatment, late recurrence, and secondary cancer development [1]. However, for most persons impacted by cancer, the ramifications are life-long, placing cancer care on a continuum beginning with prevention, risk reduction, screening, and diagnosis and leading to treatment, survivorship, and end-of-life care [4].

The cancer care continuum begins at prevention, which includes reducing risk factors and improving access to appropriate cancer screening. To aid in this endeavor, the American Society of Clinical 
Oncologists (ASCO) have suggested a patient-centered medical home for cancer care model of care delivery, bringing these preventive services into the traditionally tertiary arena, promoting enhanced communication, coordination, and accountability [4]. This model ties in preventive services with oncology specialties and pharmaceuticals. Beyond diagnosis however, treatment has changed little in the recent years. The dominant treatments for cancer continue to include surgery, radiation therapy, and systemic therapy, which may be used alone or in combination depending on cancer type and stage [1]. Furthermore, cancer care is entering an era of personalized medicine. According to the National Cancer Institute (NCI), personalized medicine is "a form of medicine that uses information about a person's genes, proteins, and environment to prevent, diagnose, and treat disease. In cancer, personalized medicine uses specific information about a person's tumor to help diagnose, plan treatment, find out how well treatment is working, or make a prognosis". Examples of such treatment include targeted therapies and tumor marking testing. Personalized medicine can also be referred to as precision medicine [5].

The shift in paradigm to personalized medicine is also seeing the emergence of various care models, which in turn has implications for the procurement and organization of resources, including facilities and space, to provide the most appropriate and state of the art cancer care. To understand the emerging models of care and implications for the cancer care industry a systematic literature was conducted. This paper addresses the findings from the literature review, and outlines the implications for the industry.

\section{Methods}

We conducted a systematic literature review, following the PRISMA checklist, to identify current trends of cancer care models and emerging trends. Our focus for this review was on clinical and management aspects of cancer care. In order to address the research purpose, search terms were created and then tested. After understanding the types of articles that would result from the search terms, additional terms were added to narrow the scope of the literature findings. From the initial search, we also determined what databases to use.

We used 15 key words, such as a combination of personalized medicine, precision medicine, patient-centered care, and team-based in terms of clinical settings. For management literature, we used other key words like a combination of team-based, team effectiveness, setting, environment, operation, care team, coordination, and cost of care. We aim to focus on articles related to new cancer care models, thus all search terms were combined with 'cancer' and 'innovation', or 'cancer' and 'new'; e.g. "personalized medicine" AND "cancer" AND "innovation". If articles were not an original study or did not address emerging models of cancer care, they were excluded.

We used PubMed for the clinical aspect and JSTOR for the management aspect to find general peer-reviewed articles including our search terms. We restricted our search to English peer-reviewed articles between January 2008 and August 2016. The date last searched is February 3, 2016. Figure 1, the PRISMA diagram, summarizes the literature review process. We collected 1,524 articles from our literature search. Once the duplicates were removed, we had 1,330 articles for further review. For the review process, we used an Excel workbook populated with general article information and abstracts. After completion of the abstract review, 1,237 articles were excluded due to being review papers or irrelevant. Next, we conducted a full literature review of 93 articles. From the full article review, 75 articles were excluded. A total of 20 articles were included, 18 from the review process ( 5 review papers) and 2 articles of interest. The findings from the 20 articles were synthesized using an Excel sheet populated with study designs and outcomes.

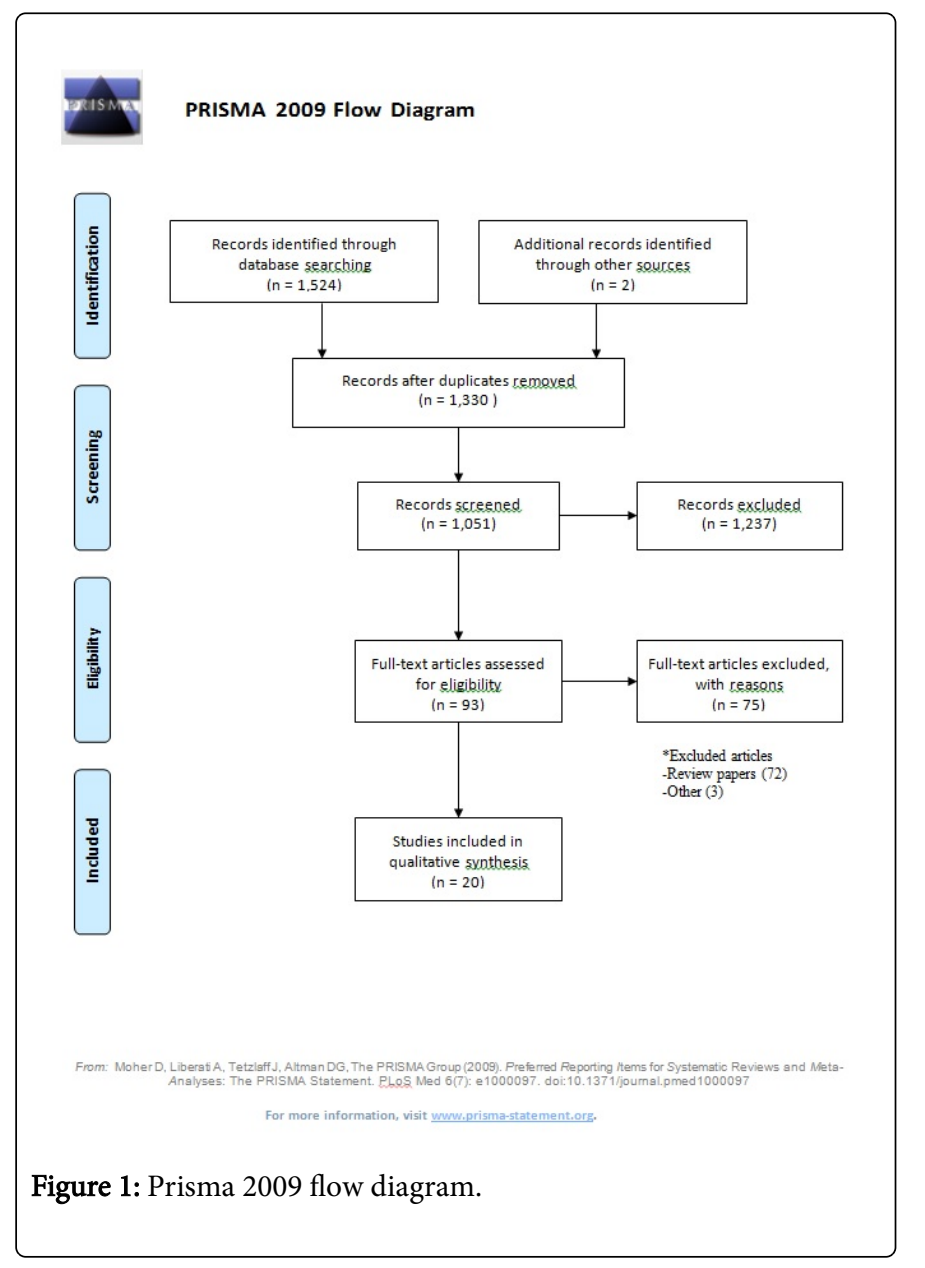

\section{Results}

Through the systematic review of literature focused on emerging models of cancer care, we identified five trends and models of care in cancer, which see to all facilitate a fully coordinated and patientcentered care environment. These five models of care are described in detail below.

\section{The hub and spoke model}

In a study by Chambers et al., it was found that there was poorer survival among patients that live further from tertiary treatment centers [6]. In an Australian study with women, some patients had to drive hundreds of kilometers to a regional center for chemotherapy and radiotherapy for post-surgery treatment [7]. Access to care is very important for any individual, but especially those with a chronic disease, such as cancer. The National Cancer Institute Community Oncology Research Program (NCORP) addresses these issues through a hub and spoke model focused on cancer research. This model of cancer research can be directly translated to a hub and spoke model focused on providing access to appropriate cancer care to all populations including geographically isolated regions. 
NCORP is a nationally recognized network of investigators, cancer care providers, academic institutions, and other relevant organizations. Throughout the United States and Puerto Rico, NCORP manages multi-site cancer clinical trials and studies in assorted populations located in community-based healthcare systems. NCORP exists to facilitate the access and opportunities that patients participate in during cancer clinical trials and cancer delivery studies. Since the participation of patients is positively correlated with the improvement of the overall cancer care, including underserved and minority, data is critical for studies that can be applicable to all groups [8].

For over thirty years, NCORP has brought research to communities through clinical research accountability, resource sharing and exchange, and partnership building. Research being located within the community grants access to larger and more diverse populations. Due to this, NCORP can accelerate accrual to clinical trials, enable feasibility testing, and increase the generalizability of study findings. NCORP also allows researchers to get together to conduct high quality clinical studies for cancer patients and those with a risk of cancer [8].

\section{NCORP consists of three major components [9]:}

- Research Bases: designed to conduct the NCORP multi-center hubs which facilitate cancer prevention, control, screening and post-treatment surveillance clinical trials and research. The bases consist of researchers with multidisciplinary expertise in clinical trials. They also provide the infrastructure for a cancer hub, including administration, data management, study operational processes and personnel, etc.

- Community Sites: a monopoly of hospitals, oncology practices, and integrated healthcare systems. The participants are involved in cancer prevention, control, screening, and post-treatment surveillance conducted through NCORP. NCORP's comparative effectiveness studies can include patients, providers, and their health systems.

- Minority/Underserved Community Sites: are like the community sites, but instead the patient populations are comprised of at least $30 \%$ racial/ethnic minorities. Minority/Underserved community sites are bringing in participants for clinical trials conducted by NCORP for treatment, imaging, and quality of life.

Khakwani et al. were the first to study a lung cancer catchment population for a surgical center and the hub and spoke model. With this model, equality care can be created by distributing resources and manpower based on need [10].

\section{The rapid learning model}

As concerns for quality of care, patient safety, and healthcare value increase, a new emerging healthcare model, referred to Rapid Learning Cancer Care Model, presents a new way of supporting effective research. Rapid Learning Cancer Care (RLCC) formulates a new learning healthcare system around three fundamental concepts. First, it aims to generate and apply the evidence that is most relevant to each patient. Second, RLCC increases the scientific discovery as an outgrowing element of the patient care. Third, there is support for the quality, assessment, and improvement of patient safety, which maximizes healthcare value [11].

Through the correct use of a full range of services, it is possible to obtain optimal quality, safety, and effectiveness in cancer care. The rapid learning healthcare system aims to 1) generate the best evidence relevant to each patient, 2) lead scientific discovery for patient care, and 3) enhance quality of care, patient safety, and healthcare value. It is important to ensure the patient-reported outcomes (PROs [ePRO=electronic $\mathrm{PRO}]$ ) are central building blocks in rapid learning healthcare. In this system, patient centeredness is at the core of healthcare and comparative effectiveness research (CER) should evaluate the impact on patients through symptoms and experiences [11].

Health care providers understand patient disease and symptoms along with preferences and concerns. In a study by Borosund et al., an interactive tailored patient assessment and communication tool called Choice was used [12]. The goal is for patients to be able to report on their experience including symptoms, problems, and priorities, and to guide clinicians in providing personalized symptom management support. Patients who used Choice experienced an increase in selfawareness and knowledge on the disease and communication improved between patient and provider. Choice helped providers to be better prepared for visits, aided in addressing difficult topics, made patients more engaged in conversations when determining how to address problems, and allowed each patient to have a voice regardless of provider personality. Choice supports the trend towards patientcentered care because it invited patients to take an active role in their care by voicing their concerns and preferences [12]. In RLCC, data collected at the individual patient level informs the most relevant care for that person. Therefore, it contributes to local evidence development and implementation of projects. At the end, it will be available for large-scale evidence synthesis, CER, and evidence implementation on the health system that maximizes healthcare value [11].

Creating an environment of affordable cancer care, the IOM recommends payment reforms and waste elimination calling for the utilization of rapid learning systems. Oncology nurses need access to timely information and opportunities to provide efficient care [13]. The cancer care team should communicate with the patients and families when creating treatment plans to ensure they align with the patient's needs, values, and preferences $[13,14]$. Patient outcomes are readily available to providers in a learning healthcare system to apply knowledge for future care [13].

The Athena Breast Health Network, an initiative of the University of California medical and cancer centers, can serve as a model for the rapid learning system. The goal is to integrate clinical care and research innovation to enhance the patient-centered breast cancer care cycle. The women enrolled in Athena provided electronic information, such as health and family history. The network implements and evaluates new methods for delivery of care, leading the way to discoveries for enhanced patient care. Athena has a cross-site and cross-disciplinary collaboration matrix to aid in the advancement of personalized medicine [15], which also relates to virtual tumor boards discussed later.

Another example of a rapid learning health care system is the ASCO's Cancer Learning Intelligence Network for Quality (CancerLinQ). CancerLinQ, a not-for-profit subsidiary of the American Society of Clinical Oncology (ASCO), analyzes real-world cancer data from any electronic source to improve quality and value as well as, clinical algorithms used in clinical decision support $[16,17]$. Physicians receive personalized decision guidance, patients are comforted in knowing they are receiving high-quality care, researchers discover new insights from the large amount of de-identified data analyzed, and payers can access metrics and tools that support quality and efficient care [16]. 
The P4 model, predictive, preventive, personalized, and participative, directly relates to the rapid learning health systems [17]. The goal of $\mathrm{P} 4$ is to transform medicine to focus on wellness, rather than disease. Systems approaches to disease, emerging technologies, and analytical tools guide the way for P4 medicine [18]. The five challenges that exist with the rapid learning and $\mathrm{P} 4$ models are: 1) collecting data properly with accurate information; 2) creating an environment of data sharing; 3 ) overcoming technical obstacles such as uniformity, incentives, oncology research complexity, and quantity of data; 4) implementing a health IT system that promotes meaningful use, directly related to step 6 with the Integrated Practice Unit Model discussed in the next section; and 5) understanding the role of government agencies like the National Institutes of Health (NIH), Centers for Medicare and Medicaid Services (CMS), and the Food and Drug Administration (FDA) [19].

\section{The integrated practice unit model for cancer}

The shift from fee-for-service to value-based care is underway. This means that changes should be made in the way that provided care aligns with new payment models. In healthcare, when we set up a goal to implement an effective plan, we need to consider how to improve value for patients. Porter and Lee describe this concept as the "health outcomes achieved that matter to patients relative to the cost of achieving those outcomes" [20]. The goal is to maximize value for patients through achieving great outcomes at the lowest cost. To accomplish this goal, Porter and Lee contend six steps that can prepare future cancer centers, all of which reinforce each other, that are outlined below [20].

Organize into Integrated Practice Units (IPUs): An IPU includes a dedicated team of clinical and non-clinical staff, and organizes the structure around the patient and their need (e.g. medical condition). An IPU aims to achieve the patient outcomes more efficiently, which results in faster treatments, better outcomes, lower costs, and improved market shares. For example, patients with chronic conditions require attention from physicians and clinicians, as well as pharmacists because their collaboration can improve patient outcomes more efficiently and effectively [20].

Sharing information for educational purposes with patients is very important because it guides the patients in making informed decisions about their care. Videos can be used to show the pros and cons of treatments, information can be provided about clinical trials, and patient navigators can be utilized during physician transitions to enhance their care experience [21]. A patient navigator is a culturally competent individual who is either a peer individual for the target population or a trained professional. Duties of a patient navigator may include peer education, appointment scheduling, appointment accompaniment, transportation, or patient support [22].

Measure outcomes and costs for every patient: Though many providers/insurers fail to track individual patient's cost and outcomes by medical condition, tracking progress of performance is vital for improvement. Quality measures rarely gauge quality, but depict compliance with practice rules. To measure outcomes that matter to patients, outcomes should be measured by medical condition and throughout the cycle of care, including the health status once treatment is completed. In addition to that, the outcomes that matter to patients should be classified into three tiers [20]:

Tier 1 (achieved health status): focusing on functional outcomes and provider performance
Tier 2 (nature of the care cycle and recovery): focusing on readmission rates, discomfort, care timeline, and delays

Tier 3 (sustainability of health): focusing on patient's and provider's perspective

Measuring patient experiences, such as perceptions and quality of life, throughout their care cycle can help improve patient-centered care [21]. Measuring these outcomes not only assists in meeting patient needs, but also helps to lower healthcare costs from improvements in functional, cycle, and long-term outcomes. Value of care can be found through measuring costs of a medical condition and tracking the expenses and specific resources for treatment. The best method to understand costs is time-driven activity-based costing (TDABC). TDABC helps providers to lower costs without affecting outcomes by better understanding opportunities, such as utilization, standardized processes, skills, and facility type. Without understanding costs, organizations are blindly deciding how to improve processes [20].

Move to bundled payments for care cycles: Fee-for-service pays based on the services a physician can provide, but the best approach is a bundled payment, which covers the overall care for a defined period of time for chronic conditions. Bundled payments encourage teamwork and improve efficiency. These types of payments may require further guidelines, such as eligibility requirements or physician accountability [20].

Using risk-adjusted reimbursements can improve standardization. Bowles et al. found that physicians tend to over treat chemotherapy patients or recommend more expensive procedure or drug options because of the incentives [21]. Providers would treat based on how much money the patient had by offering certain treatment options or withholding discussing certain treatment options [21]. By moving to bundled payments, physicians are incentivized to provide effective and efficient services because they receive one payment for that care cycle.

Integrate care delivery systems: integrated Care Delivery Systems improve value by decreasing fragmentation and duplication and enhance the types of care in each location. For true system integration, organizations should consider four factors [20]:

Define the scope of services: Organizations should determine what services can be effectively delivered and eliminate services that cannot achieve high value.

Concentrate volume in fewer locations: Providing everything a patient needs close to home or work is a good marketing technique, but it does not help improve value. Therefore, providers should concentrate care for conditions they can treat in fewer locations.

Choose the right location for each service: The goal is to deliver services where the highest value is obtained so that complexity and skill needed match the location.

Integrate care across locations: The different sites need to be networked together to meet patients' needs.

Health technology assessment (HTA) is a process used in decision making, where it translates basic research into products that can be used for personalized health care, and assesses the costs associated with the benefits of new technologies. Since cancer consists of molecular defects that affect biological processes at the cellular level and in turn affects the whole person (a systems biology disease), treatment should be based on the individual disease, which is personalizing medicine. Cancer treatment centers need molecular analysis systems that are fully automated with high-throughput, as well 
as cross-institutional electronic health records (EHRs) to fully understand the disease and family history [23]. EHRs can be used to promote multidisciplinary practice through improved informational flow and access in an organizational setting [24]. HTA can be used when implementing such systems to ensure the most effective equipment will be utilized [23].

A study in the Netherlands found that the most important imaging techniques are currently computed tomography (CT), ultrasound, and magnetic resonance imaging (MRI). After discussion, the panel came to the consensus that MRI and positron emission tomography (PET) tests will become more popular [25]. It is important to understand the scope of services the clinic will offer, but also understand what certain tests physicians are requesting.

Wait times were becoming longer, communication was lacking, and there was a shortage in employees, creating capacity problems in the Netherlands study. To address these issues, clinic hours should be properly adjusted and more staff should be hired [25]. To provide the best care, it should be understood where diagnostic services are most needed. Once noted, clinics should be built in those areas to concentrate the volume in a smaller amount of locations with the services that are most needed for that area.

Expand geographic reach: superior providers need to broaden their reach through strategic expansion to serve more patients. The first model to do this is called hub-and-spoke (seen with NCORP), which is where satellite facilities are created and the staff are employed through the parent organizations. The second model is called the emerging geographic expansions. This is when providers partner with other community or local organizations and use their facilities to provide care [20].

The Athena Breast Health Network uses a cross-site and crossdisciplinary collaboration structure to gain access to larger and more diverse patient populations [15].

Build an enabling information technology platform: if a system implements the right information technology (IT) system, it can aid in IPU teamwork. It not only enables measurement and new reimbursement approaches, but also links delivery system parts together. Value-enhancing IT platforms have six characteristics [20]:

\section{Patient-centered focus}

Standardized data definitions (diagnoses, treatments, labs)

All types of patient data incorporated

Access for all parties involved in care

Templates and expert systems for medical conditions included

System setup allows easy extraction of information

Bowles et al. included a seventh aim, coordination of care, due to handoffs being a norm for cancer patients [21]. In this study, the researchers conducted interviews with cancer care experts to discuss quality, barriers, and innovations [21,22]. Electronic medical records (EMRs) have the ability to improve communication between providers and can be utilized to provide telehealth services, improving care coordination and standardization of care $[21,24]$. It was discussed that with the use of EMRs and warning systems in the diagnostic and treatment stages, variation among providers could be reduced. EMRs can also be used to schedule patient appointments or provide remote diagnostic consultation to create timely transitions from diagnosis to treatment [21].
Multidisciplinary work can provide unity, meaning specialist communicate and collaborate to provide the best care to each patient $[23,24]$. In a study conducted by Oborn, Barrett, and Davidson, a multidisciplinary team utilized a health information technology (HIT) application to promote collaboration and coordination [24]. The system allowed surgeons to quickly check boxes during patient visits and focus on assessing lumps, and also helped nurses focus on the patient experience by building relationships with the patients. Through the use of a HIT application and multidisciplinary meetings, unity was created in patient treatment plans, transparency was promoted, and a learning environment was created for providers [24,25].

\section{The tumor board review: online/offline}

According to the NCI, a tumor board review is "a treatment planning approach in which a number of doctors who are experts in different specialties (disciplines) review and discuss the medical condition and treatment options of a patient. In cancer treatment, a tumor board review may include that of a medical oncologist (who provides cancer treatment with drugs), a surgical oncologist (who provides cancer treatment with surgery), and a radiation oncologist (who provides cancer treatment with radiation)" [26]. The tumor board is increasingly becoming "virtual", leveraging technology to bring together the multidisciplinary team required to create a care plan for the patient.

Cancer care continues to call for a multidisciplinary approach, involving different specialty experts. To facilitate this approach, tumor boards were created to have the different specialties when designing treatment plans. Tumor boards have been involved in cancer care for decades and they continue to assist the flow of treatment planning for cancer patients. Physicians come together to discuss options and treatment plans for cancer patients and make decisions on cancer care management, which improves care and coordination for the patients $[21,27]$. With different specialties involved, different ideas are brought to the table. The importance of tumor boards can be seen through the requirement of the American College of Surgeon's Commission for a cancer program to be accredited [27]. Tumor boards involve multiple disciplines to develop the best treatment plan and ideally, improve patient outcomes [28].

Virtual tumor boards exist so that the multidisciplinary approach can be continued throughout different hospitals and health systems to deliver the standard of care and improve patient outcomes and satisfaction. Not every facility or system has a virtual tumor board; currently, only those in a large metropolitan area with academic medical centers utilize these boards. To overcome the limiting aspect of tumor boards relying on a diverse and specialized local oncology department, the use of videoconference to create virtual tumor boards was developed. By including multiple health systems into developing a treatment plan, the limitation of regions no longer results in substandard cancer care. As videoconference technology is increasing, the reach of virtual tumor boards is expanding. Virtual tumor bards have been successfully implemented with widespread success and are the future of cancer care because they improve access, while maintaining feasibility and acceptability [29].

Personalized medicine requires the utilization of a multidisciplinary team of clinicians, research scientists, computer experts, and biostatisticians to provide the excellent care and achieve the best outcomes [30]. In a UK survey, $81 \%$ of multidisciplinary team members agreed that job satisfaction was improved and $90 \%$ reported that clinical decision making, patient care coordination, quality of care, 
evidence-based treatment decisions, and treatment all improved through effective multidisciplinary teamwork [31].

In a study conducted in Los Angeles and Detroit by Katz et al. on breast cancer patients, it was found that about $32.9 \%$ of these patients received multidisciplinary physician communication with providers associated with teaching programs versus $16.5 \%$ with providers associated with non-teaching programs [32]. In a Singapore study looking at women with breast cancer, the screening program, BreastScreen Singapore, adopted several factors including weekly multidisciplinary review sessions, to improve breast cancer detection rates [33]. It was discussed that there are logistical and cost barriers to implement collaboration. It was reported that more specialized surgeons participated in a greater amount of collaborative communication because they were more willing and/or had greater opportunities to invest in same day appointments and weekly tumor board meetings. Surgeons with a smaller breast cancer patient load would better benefit from virtual tumor boards $[32,33]$.

Brier Scores were used to determine if physicians could provide a better prediction of survival at 6 months in a study conducted by Kee, Owen, and Leathem in Northern Ireland [34]. It was found that group discussion did not significantly change accuracy of the prediction for an individual provider, but individuals and the team both performed better after discussion. Clinicians also expressed feeling more confident after discussing patient cases with other providers [34].

A virtual tumor board is a multidisciplinary team that discusses HTA to implement the proper systems. Access to EHRs is also necessary when discussing patient cases. Both, HTA and EHRs, create an environment of personalized medicine, better treatment plans, and improved outcomes (refer to Integrated Practice Unit section).

\section{A tiered model of psychosocial care}

Due to cancer diagnosis causing a great amount of emotional distress, psychosocial intervention should be promoted to patients. Frequent screening can be beneficial to ensure services are being utilized (Hutchison et al., 2006). By providing psychosocial treatment, cancer patients are being guided to have positive long-term outcomes with improved quality of life through an enhanced ability to cope with the disease and treatment, build trust in the providers, and improve compliance $[6,35]$. The active behavioral approach allows providers to better understand the nonverbal needs, preferences, and expectations of patients. These are expressed through nonverbal body language cues, but once recognized, they should be addressed through verbal psychosocial support [35].

This model suggests triaging cancer patients for psychosocial distress and matching their level of distress to the level of care. There are five levels to the model, where the first is generic care and the last is more specialized care [36]:

Level 1, universal care, is for any cancer patient or caregiver that has mild distress. In this level, general information is provided about treatment and potential side effects and can be conducted via a primary care team, print materials, or cancer call lines.

Level 2, supportive care, is for those suffering with mild to moderate distress. Levels 2 provide psycho-education with emotional support, and peer and decision support. Care options include cancer call lines, hospital or community-based psycho-education, and peer support groups. Topics discussed include decision making for treatment, communicating with physicians, coping, and problem solving.
Level 3, extended care, is for those with strictly moderate distress and provides time-limited, semi-structured care concentrated on cancer-specific issues. In this level, treatment can be conducted via group/individual therapy or tertiary psychology clinics. The topics for level 3 include adjustment, stress management, communication, coping, and problem solving skills.

Level 4, specialist care, is for those suffering with moderate to severe distress. Treatment is provided through couple or individual therapy with a psychologist, psychiatrist, sex or grief therapist. Pharmacotherapy is also an option through a primary care provider or psychiatrist. Topics include mood and anxiety disorders, and relationship problems.

Level 5, acute care, is for those with strictly severe distress. This level deals with multiple problems and can be provided through a variety of professionals. A mental health team can be created to care for this patient, which could include a psychologist, psychiatrist, family therapist, and multidisciplinary mental health services. Topics for level 5 include suicide ideation, couple problems, family issues, and personality problems.

\section{Discussion}

The 2016 report on the State of Cancer Care in America highlights a number of key issues currently facing clinical oncology, including: increasing complexity of care delivery, remaining gaps in insurance coverage, rising costs of cancer care, issues with access to care, and inconsistent adoption for best practices [4]. The increased prevalence, longer continuum, and rapidly changing policy environment, of cancer, has resulted in the new care models discussed, which could have significant implications for health systems- and impact their process, policy, technology and facility decisions.

Hub and spoke model: System wide real estate decisions to place community centers and regional hubs with access to research bases leveraging community partnerships and cloud-based collaborations

Rapid learning model: Easy and ubiquitous access to data, across the system, with the seamless integration of technology and appropriate space to ensure that care delivery is enhanced.

Integrated practice unit model: Rethinking the traditional service lines and conventional facility layouts, and consider new emerging roles like the patient navigator. Also rethinking the extensive retail driven movement to have too many access points which do not always add value (because they don't have the required volumes to provide expert specialty care).

Tumor board review: the workplace and technologies in cancer clinics are slated for a complete transformation. Multidisciplinary teams will need to be accommodated which implies that the traditional physician and specialist offices may be a thing of the past. Additionally the accommodation of increasing video conferencing and data sharing via a range of devices will create a tiered approach to work- where traditional room types will be challenged and technology will become increasingly embedded in the facilities. This will also have implications on the team structures and make virtual collaboration a norm.

Tiered model of psychosocial care: the variety of appropriate care options have to be accessible for the patients who need care, such as information, peer support, psychological and psychiatric care [36]. Policies, processes, technologies and space have to be focused on a psychosocial approach that promotes healing. 
A key takeaway from this literature review was that in addition to the changes we will definitely see in technology, operational processes, and system wide policies; we will also see a transformation of cancer care facilities. Unfortunately, the implications emerging models on facility design have not been widely examined. The research team is working on exploring the brick and mortar implications of the emerging care models through a series of focus groups and key informant interviews to create a qualitative base for further investigations. These findings will be shared in an upcoming publication. Another limitation includes only examining the implications for facilities design and not yet studying implementation outcomes. The hope is future research publications will cover implementation methodologies and outcomes.

\section{Conclusion}

The emerging models of cancer care show promise for developing systemic solutions to deliver personalized, quality medicine in a fully coordinated environment. The hub and spoke model, rapid learning model, integrated practice model, tumor board review model, and tiered model of psychosocial care could fundamentally transform what cancer care looks like in the future. Though not greatly studied, this will also have significant implications on facility design including layout, location, embedded technology, and sensory experience. This warrants further study by interdisciplinary teams.

\section{Funding}

This is research was funded by a National Science Foundation (NSF) Industry-University Cooperative Research Centers Program (IUCRC) agreement between NSF Center for Health Organization Transformation (CHOT) and HKS.

\section{Authors' Contributions}

Kash made substantial contributions in designing the research approach and manuscript development. McKahan and Mack were involved in the systematic literature search, data analysis, and development of the draft manuscript. Nanda made contributions to discussion and implication section of this study. All authors have been involved in drafting and revising the manuscript.

\section{References}

1. Cancer Treatment \& Survivorship Facts \& Figures (2016) American Cancer Society.

2. Cancer Facts \& Figures (2016) American Cancer Society.

3. Cancer Trends Progress Report (2016) National Cancer Institute.

4. The State of Cancer Care in America (2016) American Society of Clinical Oncology.

5. Types of Treatment (2016) National Cancer Institute.

6. Chambers SK, Meng X, Youl P, Aitken J, Dunn J, et al. (2012) A five-year prospective study of quality of life after colorectal cancer. Qual Life Res 21: 1551-1564.

7. Wainer J, Willis E, Dwyer J, King D, Owada K (2016) The treatment experiences of Australian women with gynaecological cancers and how they can be improved: A qualitative study. Reprod Health Matters 20: 38-48.

8. National Cancer Institute Community Oncology Research Program (2016) National Cancer institute.

9. National Cancer Institute Community Oncology Research Program: NCORP Structure (2016) National Cancer Institute.
10. Khakwani A, Rich AL, Powell HA, Tata LJ, Stanley RA, et al. (2015) The impact of the 'hub and spoke' model of care for lung cancer and equitable access to surgery. Thorax 70: 146-151.

11. Abernethy AP, Ahmad A, Zafar SY, Wheeler JL, Reese JB, et al. (2010) Electronic patient-reported data capture as a foundation of rapid learning cancer care. Med Care 48: S32-38.

12. Borosund E, Ruland CM, Moore S, Ekstedt M (2014) Nurses' experiences of using an interactive tailored patient assessment tool one year past implementation. Int J Med Inform 83: e23-34.

13. Ferrell B, McCabe MS, Levit L (2013) The Institute of Medicine report on high-quality cancer care: implications for oncology nursing. Oncol Nurs Forum 40: 603-609.

14. Louie AV, Palma DA, Dahele M, Rodrigues GB, Senan S (2014) Management of early-stage non-small cell lung cancer using stereotactic ablative radiotherapy: controversies, insights, and changing horizons. Radiother Oncol 114: 138-147.

15. Elson SL, Hiatt RA, Anton-Culver H, Howell LP, Naeim A, et al. (2013) The Athena Breast Health Network: developing a rapid learning system in breast cancer prevention, screening, treatment, and care. Breast Cancer Res Treat 140: 417-425.

16. Cancerlinq (2017) American Society of Clinical Oncology.

17. Shaikh AR, Butte AJ, Schully SD, Dalton WS, Khoury MJ, et al. (2014) Collaborative biomedicine in the age of big data: the case of cancer. J Med Internet Res 16: e101.

18. Hood L, Friend SH (2011) Predictive, personalized, preventive, participatory (P4) cancer medicine. Nat Rev Clin Oncol 8: 184.

19. Kean MA, Abernethy AP, Clark AM, Dalton WS, Pollock BH, et al. (2012) Achieving data liquidity in the cancer community: proposal for a coalition of stakeholders (discussion paper). Institute of Medicine.

20. Porter ME, Lee TL (2013) The strategy that will fix healthcare. The Harvard Business Review.

21. Aiello Bowles EJ, Tuzzio L, Wiese CJ, Kirlin B, Greene SM, et al. (2008) Understanding high-quality cancer care: a summary of expert perspectives. Cancer 112: 934-942.

22. Fashoyin-Aje LA, Martinez KA, Dy SM (2012) New patient-centered care standards from the commission on cancer: opportunities and challenges. J Support Oncol 10: 107-111.

23. Becla L, Lunshof JE, Gurwitz D, Schulte in den Ba"umen T, Westerhoff $\mathrm{HV}$, Lange BMH, Brand A. Health technology assessment in the era of personalized health care. International J of Technology Assessment in Health Care 27: 118-126.

24. Oborn E, Barrett M, Davidson E (2011) Unity in diversity: electronic patient record use in multidisciplinary practice. Information Systems Research 22: 547-564.

25. Postma TJ1, Alers JC, Terpstra S, Zuurbier A (2006) The future of imaging techniques for cancer patients in The Netherlands : A Delphi study. Eur J Health Econ 7: 117-122.

26. NCI Dictionary of Cancer Terms (2016) National Cancer Institute.

27. Keating NL, Landrum MB, Lamont EB, Bozeman SR, Shulman LN, et al. (2013) Tumor boards and the quality of cancer care. J Natl Cancer Inst 105: 113-121.

28. Stevenson MM, Irwin T, Lowry T, Ahmed MZ, Walden TL, et al. (2013) Development of a virtual multidisciplinary lung cancer tumor board in a community setting. J Oncol Pract 9: e77-80.

29. Marshall CL, Petersen NJ, Naik AD, Vander Velde N, Artinyan A, et al. (2014) Implementation of a regional virtual tumor board: a prospective study evaluating feasibility and provider acceptance. Telemed J E Health 20: 705-711.

30. Diamandis M, White NMA, Yousef GM (2010) Personalized medicine: making a new epoch in cancer patient management. Mol Cancer Res 8: 1175-1187.

31. Multidisciplinary team members' views about MDT working: results from a survey commissioned by the National Cancer Action Team (2017) National Cancer Action Team. 
Citation: Kash B, McKahan M, Mack S, Nanda U (2017) Systematic Review of Emerging Models of Cancer Care: Implications for the Health Industry. J Integr Oncol 6: 196. doi:10.4172/2329-6771.1000196

Page 8 of 8

32. Katz SJ, Hawley ST, Morrow M, Griggs JJ, Jagsi R, et al. Coordinating cancer care: patient and practice management processes among surgeons who treat breast cancer. Med Care 48: 45-51.

33. Jara-Lazaro AR, Thilagaratnam S, Tan PH (2010) Breast cancer in Singapore: some perspectives. Breast Cancer 17: 23-28.

34. Kee F, Owen T, Leathem R (2007) Offering a prognosis in lung cancer: when is a team of experts an expert team? J Epidemiol Community Health 61: 308-313.
35. Bencová V (2013) [Communication as a part of the supportive treatment in cancer care]. Klin Onkol 26: 195-200.

36. Hutchison SD, Steginga SK, Dunn J (2006) The tiered model of psychosocial intervention in cancer: a community cased approach. Psychooncology 15: 541-546. 